\title{
Coexistencia infrecuente de la enfermedad de Tay-Sachs, coartación aórtica y reflujo vesicoureteral de grado $\mathrm{V}$ Rare coexistence of Tay-Sachs disease, coarctation of the aorta and grade $V$ vesicoureteral reflux
}

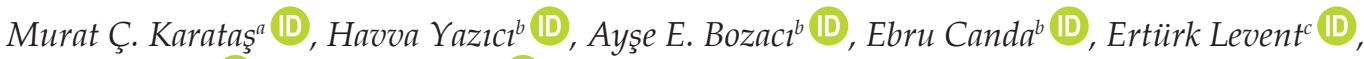 \\ Sema K. Uçarb (D), Mahmut Çokerb (D)
}

\section{RESUMEN}

La enfermedad de Tay-Sachs es una enfermedad metabólica hereditaria neurodegenerativa. Existen cuatro tipos según el inicio de los síntomas clínicos: infantil, infantil de inicio tardío, juvenil y adulto. El tipo infantil tiene el peor pronóstico. Recientemente, se describieron diferentes anomalías que acompañan a los trastornos metabólicos e influyen en el pronóstico.

Presentamos el caso de un lactante conenfermedad de Tay-Sachs junto con coartación aórtica y reflujo vesicoureteral bilateral (RVU) de grado V. Se realizó el seguimiento del paciente en el consultorio externo de Cardiología Pediátrica. En la ecografía abdominal, se observó ectasia pielocalicial, y se detectó reflujo vesicoureteral bilateral de grado $\mathrm{V}$ en la cistouretrografía miccional. No se ha informado previamente la coexistencia de estas anomalías. Este caso pone de manifiesto que no se deben subestimar las anomalías del examen neurológico en los pacientes con una cirugía cardíaca reciente, porque podría perderse la oportunidad de diagnosticar enzimopatías congénitas.

Palabras clave: enzimopatías congénitas, trastorno de depósito lisosomal, gangliosidosis monosialogangliósido 2 (GM2), anomalías congénitas, coartación aórtica.

http: / / dx.doi.org/10.5546/ aap.2022.e25

Texto completo en inglés:

http: / / dx.doi.org/10.5546/ aap.2022.eng.e25

Cómo citar:Karataş MC, YazıcıH, Bozacı AE, CandaE, etal.Coexistencia infrecuente de la enfermedad de Tay-Sachs, coartación aórtica y reflujo vesicoureteral de grado V. Arch Argent Pediatr 2022;120(1):e25-e28.

a. Departamento de Pediatría.

b. División de Metabolismo y Nutrición en Pediatría.

c. División de Cardiología Pediátrica.

Ege Üniversitesi, Esmirna, Turquía.

Correspondencia:

Murat Ç. Karataş: muratcaglarkaratas@hotmail.com

Financiamiento: Ninguno.

Conflicto de intereses: Ninguno que declarar.

Recibido: $11-12-2020$

Aceptado: 29-7-2021

\section{INTRODUCCIÓN}

La enfermedad de Tay-Sachs (TSD) es una enfermedad metabólica recesiva autosómica con acumulación de gangliósidos GM2 en los lisosomas debido a una deficiencia de la enzima $\beta$-hexosaminidasa A (HEXA). Causa neurodegeneración y se clasifica en cuatro tipos clínicos diferentes: infantil, infantil de inicio tardío, juvenil y adulto. ${ }^{1}$

El tipo infantil de la enfermedad tiene el peor pronóstico clínico. Los primeros síntomas de este tipo, como debilidad muscular e hipotonía, aparecen en torno a los 4 a 6 meses. La macrocefalia y la respuesta exagerada de sobresalto en la exploración física son signos importantes. Habitualmente, se produce la muerte debido a una neumonía por aspiración antes de los 3 años. ${ }^{2}$ Los tipos de presentación infantil de inicio tardío y juvenil suelen verse entre los 2 y los 10 años. En el tipo adulto, es notable la existencia de psicosis junto con síntomas piramidales y extrapiramidales (distonía, asteatosis, ataxia). ${ }^{1,2}$ En los pacientes cuya anamnesis y exploración física arrojan resultados sospechosos, el diagnóstico de la enfermedad de Tay-Sachs se establece por la actividad anormalmente baja de la enzima HEXA en las pruebas enzimáticas y la identificación de variantes patógenas bialélicas en el gen HEXA en las pruebas genéticas moleculares. Hasta el momento, no existe un tratamiento que cure la enfermedad de Tay-Sachs. La enfermedad de Tay-Sachs tiene una frecuencia de 1/320 000 en la población general; en los judíos askenazi, la frecuencia es de $1 / 3900 .^{3}$

La frecuencia de las cardiopatías congénitas es aproximadamente del $0,8 \%$ al $1,2 \%$ en los nacidos vivos. ${ }^{4}$ La coartación aórtica (CoAo) es una anomalía obstructiva congénita del lumen aórtico que se observa en el $5 \%$ al $7 \%$ de todas las cardiopatías congénitas y tiene una tasa de mortalidad elevada en los pacientes no diagnosticados. ${ }^{5}$ 
Las enfermedades metabólicas hereditarias traen aparejadas anomalías cardíacas y renales. Por ejemplo, en algunos defectos de oxidación de los ácidos grasos, se observan síntomas de miocardiopatía grave. Los trastornos en el metabolismo de la carnitina, la enfermedad de Pompe, la enfermedad de Fabry, la gangliosidosis GM1, un defecto congénito en la glucosilación y algunas enfermedades mitocondriales son otras enfermedades metabólicas que pueden producir efectos cardíacos. La aciduria glutárica de tipo II puede causar riñones microquísticos. Los pacientes con diagnóstico de galactosemia e intolerancia hereditaria a la fructosa que no cumplen con el tratamiento farmacológico pueden presentar tubulopatía proximal e insuficiencia renal. La tirosinemia de tipo 1 puede producir una tubulopatía, y la cistinosis puede causar disminución de la tasa de filtración glomerular e insuficiencia renal terminal. ${ }^{6}$ Sin embargo, previamente no se han descripto las anomalías cardíacas y renales relacionadas con la enfermedad de Tay-Sachs.

\section{A PROPÓSITO DE UN CASO}

El bebé nació por cesárea en la semana 38 de embarazo, con un peso al nacer de 3340 gramos. La madre era una mujer sana que se había casado con un primo hermano. Se hospitalizó al paciente en la unidad de cuidados intensivos neonatales debido a una dificultad respiratoria que comenzó a los 8 días de vida. Durante el seguimiento, se detectaron taquipnea, un soplo sistólico $3 / 6$ y aumento de la presión arterial en las extremidades superiores en la medición de la presión arterial en las cuatro extremidades. También se identificó un importante estrechamiento de la aorta (gradiente de $50 \mathrm{mmHg}$ ) en la ecocardiografía. En la angioTC torácica, se observó una comunicación interauricular (CIA) de tipo ostium secundum de $5 \mathrm{~mm}$ de diámetro y coartación aórtica distal (Figura 1). Por lo tanto, se operó al paciente a

Figura 1. AngioTC torácica. La aorta estaba hipoplásica en la parte distal al cayado aórtico, dando origen a la segunda rama. La aorta ascendente media $11 \mathrm{~mm}$; el cayado aórtico proximal, 9,1 mm (proyección 4 en 3D); y el segmento hipoplásico distal medía $5 \mathrm{~mm}$ de diámetro (proyección 1 en 3D). En la aorta, se observó coartación avanzada distal en el origen de la arteria subclavia izquierda (flecha). El diámetro se observa disminuido a 1,1 mm (proyección 2 en 3D). El diámetro de la aorta descendente era de 7,5 $\mathrm{mm}$ (proyección 3 en 3D).

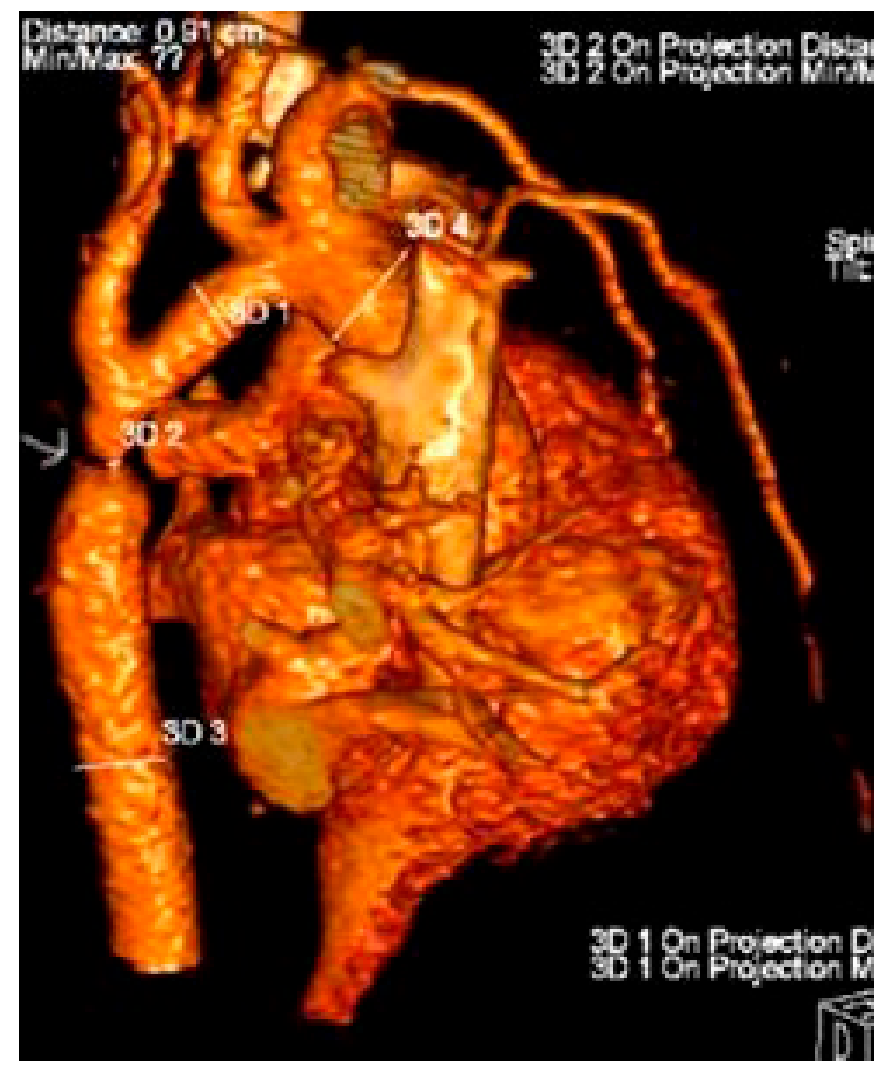


los 24 días de vida. Se extirpó el segmento de la coartación y se realizó una anastomosis términoterminal.

Se realizó el seguimiento del paciente en el consultorio externo de Cardiología Pediátrica. A los 9 meses de edad, se hospitalizó al paciente por retraso mental y motor. En la ecografía, se detectó ectasia pielocalicial (diámetro AP: $6 \mathrm{~mm}$ a la derecha, $5 \mathrm{~mm}$ a la izquierda), y se halló reflujo vesicoureteral (RVU) bilateral de grado V en la cistouretrografía miccional. Los análisis de micromatriz y FISH (hibridación in situ con fluorescencia), realizados debido a la coartación aórtica y la patología renal concomitante, fueron normales.

El paciente tenía una regresión del desarrollo neuromotor, y se detectó de forma bilateral una mancha roja en la mácula durante el examen visual. En la exploración física, el paciente estaba consciente. Tenía poco control de la cabeza. No podía sentarse sin ayuda, sus movimientos eran lentos y tenía una disminución del tono muscular. Se identificó hiperacusia. Con respecto a las pruebas analíticas, los análisis bioquímicos de la sangre y los análisis de líquido cefalorraquídeo (proteína, glucosa, ácido láctico) fueron normales. En la resonancia magnética (RM) y la resonancia magnética espectroscópica (RME), no se detectaron anomalías significativas. Para la evaluación neurofisiológica, se realizaron una prueba de potenciales evocados auditivos de tronco cerebral y una electromiografía, cuyos resultados fueron normales. Sin embargo, se observó un defecto de conducción parcial bilateral en los potenciales evocados visuales.

Se evaluó al paciente a través de los antecedentes y los hallazgos de la exploración física por la sospecha de enfermedades neurometabólicas. Se detectaron niveles considerablemente bajos de la enzima HEXA $(0,5 \mathrm{nmol} / \mathrm{ml} /$ hora en sangre desecada, normal: 7-70). En el análisis molecular, se identificó una mutación homocigótica de c.1361G>A (p.Gly454Asp) en el gen HEXA. La mutación se confirmó mediante la secuenciación de Sanger de los padres. Ambos padres eran heterocigóticos.

\section{DISCUSIÓN}

El nombre Tay-Sachs surge por el oftalmólogo británico Warren Tay, que definió por primera vez las manchas rojas en la retina (uno de los síntomas de la enfermedad) en 1881, y el neurólogo estadounidense Bernard Sachs, que informó por primera vez los cambios en el estado celular durante la enfermedad en 1887. La enfermedad es más frecuente en Europa Oriental y en los judíos askenazi. ${ }^{7}$ En los niños con esta enfermedad, los primeros meses de vida son normales; sin embargo, después del sexto mes (aproximadamente), aparecen síntomas y la enfermedad causa pérdida progresiva de la inteligencia, demencia, evitación del contacto visual, respuesta exagerada de sobresalto, hipoacusia, disfagia, pérdida de la visión, manchas rojas en la retina y espasticidad. En el primer año, suelen presentar convulsiones mioclónicas. También es posible que tengan convulsiones focales y generalizadas. La mayoría de los pacientes con la enfermedad de TaySachs mueren antes de los 5 años. Como no se dispone de un tratamiento que pueda curar esta enfermedad, el tratamiento de apoyo es fundamental. Estos tratamientos incluyen una dieta adecuada y métodos que permitan mantener las vías aéreas abiertas.

La enfermedad de Tay-Sachs de inicio tardío, que es un tipo más infrecuente, se presenta cuando el paciente tiene poco más de 20 años con síntomas como dificultad para caminar y trastornos neurológicos progresivos. ${ }^{8}$ En la actualidad, se están investigando nuevos modelos de tratamiento. Se han realizado estudios clínicos sobre la terapia de reducción de sustrato con miglustat y el trasplante de células madre hematopoyéticas. También hay métodos de genoterapia que están en desarrollo. ${ }^{9}$ Se demostró que el uso de la terapia de reducción de sustrato (miglustat) previene la acumulación de gangliósidos GM2 en el cerebro de modelos murinos con la enfermedad de Tay-Sachs. ${ }^{10}$ Sin embargo, en algunas presentaciones de casos se informa que el uso de miglustat no detuvo el progreso de la disfunción neurológica. ${ }^{11}$

La coartación aórtica tiene altas tasas de mortalidad en los pacientes no diagnosticados. Suele ser esporádica y es más frecuente en los hombres. La coartación aórtica se divide en dos grupos: crítica (aproximadamente el $60 \%$ de todos los casos), que muestra síntomas en el transcurso de los primeros dos meses de vida y, si no se trata, causa la muerte, y asintomática, en la que los síntomas, junto con la hipertensión en las extremidades superiores, aparecen en años posteriores. Los pacientes con coartación aórtica tienen un alto riesgo de complicaciones y muerte. Si bien en el $50 \%$ al $70 \%$ de los casos se observa la presencia de una válvula aórtica bicúspide, entre las otras anomalías congénitas 
concomitantes se incluyen hipoplasia tubular del cayado aórtico, conducto arterial persistente, comunicación interventricular y comunicación interauricular. ${ }^{5,12}$ En el electrocardiograma, se ven signos de hipertrofia ventricular derecha, contrariamente a las expectativas. Estudios recientes en Escandinavia indicaron que, como mínimo, el $50 \%$ de los recién nacidos con coartación aórtica reciben el alta sin un diagnóstico. Según otro estudio, el $27 \%$ de los pacientes con coartación aórtica fallecieron en un promedio de 17 días sin diagnóstico. ${ }^{13}$ La forma más sencilla de diagnosticar la coartación aórtica es evaluando el pulso femoral de todos los recién nacidos entre el tercer y el séptimo día de vida, lo que permite derivarlos al hospital para realizar más exámenes cuando sea necesario. ${ }^{12}$

Nuestro paciente, que tenía reflujo vesicoureteral de grado $\mathrm{V}$, coartación aórtica y comunicación interauricular de tipo ostium secundum identificados en el período neonatal, recibió el diagnóstico genético y enzimático de la enfermedad de Tay-Sachs como resultado de los estudios diagnósticos realizados, además de los hallazgos oftalmológicos y los síntomas neurológicos en el seguimiento.

Se han informado algunos casos de simultaneidad de otras enfermedades y la enfermedad de Tay-Sachs. Acar S. y col., presentaron a dos pacientes con confirmación genética de la enfermedad de Tay-Sachs a quienes, durante el seguimiento, se les detectó pubertad precoz central. ${ }^{14}$ Ahmed NR. y col., presentaron un caso de enfermedad de TaySachs infantil con un coloboma coriorretiniano unilateral..$^{15}$ Sin embargo, en la revisión de las publicaciones, no se ha descrito previamente la coexistencia de la enfermedad de Tay-Sachs y la coartación aórtica. Aún no se ha aclarado si esta coexistencia es una coincidencia o si el metabolismo de los gangliósidos cumple alguna función. Hacemos hincapié en que no se deben subestimar las anomalías del examen neurológico en los pacientes con una cirugía cardíaca reciente. De lo contrario, podría perderse la oportunidad de diagnosticar enzimopatías congénitas.

\section{REFERENCIAS}

1. Pastores GM, Hughes DA. Non-neuronopathic lysosomal storage disorders: disease spectrum and treatments. Best Pract Res Clin Endocrinol Metab. 2015; 29(2):173-82.

2. Pastores GM, Maegawa GHB. Clinical neurogenetics: neuropathic lysosomal storage disorders. Neurol Clin. 2013; 31(4):1051-71.

3. Lew RM, Burnett L, Proos AL, Delatycki MB. Tay-Sachs disease: current perspectives from Australia. Appl Clin Genet. 2015; 8:19-25.

4. Marelli AJ, Mackie AS, Ionescu-Ittu R, Rahme E, Pilote L. Congenital heart disease in the general population: changing prevalence and age distribution. Circulation. 2007; 115(2):163-72.

5. Aboulhosn J, Child JS. Left ventricular outflow obstruction: subaortic stenosis, bicuspid aortic valve, supravalvar aortic stenosis, and coarctation of the aorta. Circulation. 2006; 114(22):2412-22.

6. Levy PA. Inborn errors of metabolism; part 1 overview. Pediatr Rev. 2009; 30(4):131-7.

7. Fernandes JA Filho, Shapiro BE. Tay-sachs disease. Arch Neurol. 2004; 61(9):1466-8.

8. VanierMT, CaillaudC.Disorders of sphingolipid metabolism and neuronal ceroid-lipofuscinoses. En: Saudubray JM, van den Berghe G, Walter JH (eds). Inborn Metabolic Diseases. Heidelberg: Springer; 2012. Págs.555-77.

9. Solovyeva VV, Shaimardanova AA, Chulpanova DS, Kitaeva KV, et al. New Approaches to Tay-Sachs Disease Therapy. Front Physiol. 2018; 9:1663.

10. Bembi B, Marchetti F, Guerci VI, Ciana G, et al. Substrate reduction therapy in the infantile form of Tay-Sachs disease. Neurology. 2006; 66(2):278-80.

11. Maegawa GHB, Banwell BL, Blaser S, Sorge G, et al. Substrate reduction therapy injuvenile GM2 gangliosidosis. Mol Genet Metab. 2009; 98(1-2):215-24.

12. Hoffman JI. The challenge in diagnosing coarctation of the aorta. Cardiovasc J Afr. 2018; 29(4):252-5.

13. Chang RKR, Gurvitz M, Rodriguez S. Missed diagnosis of critical congenital heart disease. Arch Pediatr Adolesc Med. 2008; 162(10):969-74.

14. Acar S, Arslan N, Paketçi A, Okur TD, et al. Presentation of central precocious puberty in two patients with TaySachs disease. Hormones (Athens). 2018; 17(3):415-8.

15. Ahmed NR, Tripathy K, Kumar V, Gogia V. Choroidal coloboma in a case of Tay-Sachs disease. Case Rep Ophthalmol Med. 2014; 2014:760746. 